\title{
VME insertion device control at the Advanced Photon Source
}

\author{
M. Smith, ${ }^{\text {a) }}$ M. Ramanathan, J. Grimmer, and M. Merritt \\ Advanced Photon Source, Argonne National Laboratory, 9700 South Cass Avenue, Argonne, Illinois 60439
}

(Presented on 22 August 2001)

\begin{abstract}
The Advanced Photon Source (APS) currently has 29 insertion devices (IDs) installed and operating. The need to remotely diagnose and correct problems has become increasingly important. This has been accomplished through the development of a new control system with greatly enhanced input/output (I/O) capabilities specifically targeted to this control task. The system features a custom VME control card and three rack-mounted interface chassis for ID control, encoder interface, and motor drive shutdown. The card provides device interlocks, limit switch logic, motor axis selection, digital I/O, and status feedback. This VME insertion device control was designed to operate with an eight-axis intelligent motor controller and a stepper-motor drive that accepts step and direction inputs. The front panel of the card has two connectors for all of the control signals for the stepper-motor drives. There is a third connector for the ID limit switch inputs and the emergency stop circuit, and a fourth connector provides 23 bits of digital outputs and 16 bits of digital inputs. Light-emitting diodes indicate which motions are inhibited by the limit switch logic. An experimental physics industrial control system (EPICS) (http://www.APS.ANL-GOV/ EPICS) device driver was developed to access all the registers on the VME control card. Using standard EPICS records, the insertion device status can be viewed remotely. This minimizes downtime for APS ID beamline users by allowing faster resolution of any problems preventing a user from operating the insertion device. This new insertion device control has been in use at the APS since July of 1999. The design features of the control system and rationale for them will be presented, along with our experience in building, testing, installing, and operating the control system. () 2002 American Institute of Physics. [DOI: 10.1063/1.1420757]
\end{abstract}

\section{INTRODUCTION}

Past operating experience with the insertion device (ID) control systems at the Advanced Photon Source (APS) has led to the development of an ID control that would allow both remote diagnostics as well as the ability to quickly identify and repair many of the operational problems that may occur. These problems usually involve the device motion, such as hitting limit switches or motor drive problems. The control system fully utilizes feedback features of the many components that comprise the system and that of the ID. The design of this control system was based on operational needs and minimum user downtime.

\section{DESCRIPTION}

The VME ID control is a full-height, double-wide, A16/ D16 (16 bit) register-based VME card incorporating throughhole integrated circuit technology for ease of assembly. The front panel contains one 40-pin condo header, two D-subminiature 9-pin (DB9) connectors, and six lightemitting diodes (LEDs) to show the device status. One of the 40-pin headers contains all of the limit switch connections to the ID control interface while the other contains all of the digital inputs and outputs. The two DB9 connectors have signals that interface to each of the two 12 A Superior Electric motor drives that are used to control the device motion.

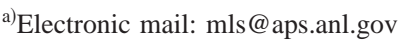

The board occupies six contiguous addresses in the VME short address space, the location dependent upon how the base address switches are set. Only the upper ten bits of the 16 bit address bus are used for address selection. The control system block diagram is shown in Fig. 1.

The ID control card was designed for a two-stepper motor insertion device that incorporates 12 individually wired limit switches. All of the IDs' limit switch and emergency stop signals are connected to the control system via four 35-pin connectors on the rear panel of the ID interface chassis. This allows connection to one of the VME card's 40-pin header connectors located on the front panel. There are eight minimum and four maximum limit switches. Of the eight minimum switches, four are hard wired in series to a rackmounted motor drive shutdown chassis. When any one of these four limit switches is hit, the input power to the stepper-motor drives will be shut off via a solid-state relay. These four limit switches contact the smallest part of the ID vacuum chamber and are considered the control system's last resort to keep the jaws of the device from coming in contact with the vacuum chamber. The other four minimum and the four maximum limit switches provide feedback to the insertion device control board. This is where the appropriate logic is performed before passing this limit information back to the motor controller.

The limit switch logic is set up to prevent damage to both the ID magnet array and the vacuum chamber of the storage ring. The reasoning for the limit switch logic is due to the placement of the drive motors and mechanically 


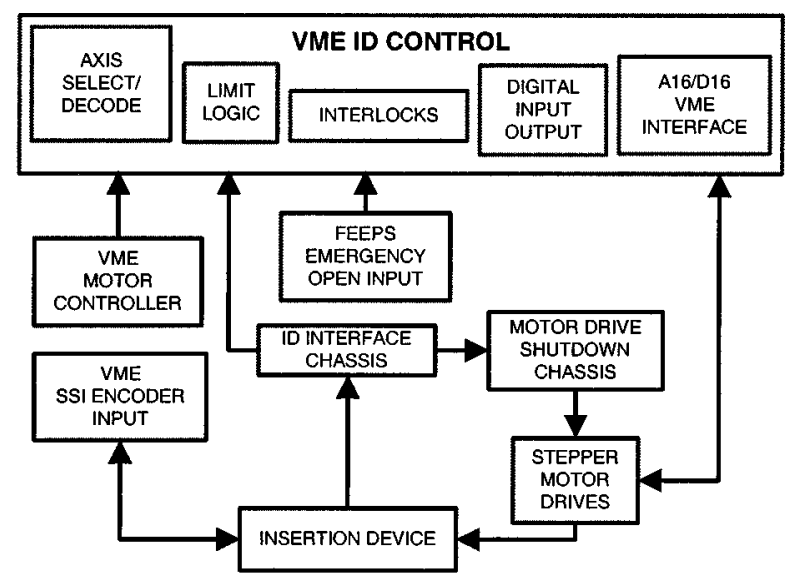

FIG. 1. ID control system block diagram.

coupled ID jaws ${ }^{1,2}$ in relation to the two ends of the jaws. This logic tells the motor controller which motions to inhibit and is displayed by four LEDs located on the VME front panel. The jaws of the ID are driven by two motors, one motor controlling each end of the device. With this in mind, the limit logic is set up such that if a minimum gap limit switch is hit, the gap may not be closed any further at the same end of the device and may not be opened at the opposite end of the device. If a maximum gap limit switch is hit, the gap may not be opened any further at the same end of the device and may not be closed any further at the opposite end of the device. With this logic, it is possible for the ID to hit the minimum gap limit switches at both ends of the device, which would inhibit all motion. A provision has been made to overcome this problem. Using standard EPICS records, a register bit can be set to allow motion in only the open direction. This bit can only be set when the minimum gap limits at both ends of the device have been hit. Once any one of these minimum limit switches is not hitting the vacuum chamber, this bit is automatically reset, at which time the normal limit switch logic will apply. There is also a register bit and a front panel LED that will indicate when a limit switch error has occurred. This error condition has been defined as a condition that is mechanically impossible for the ID to accomplish unless something is broken; e.g., a minimum and maximum gap limit switch that is hitting at the same end of the ID at the same time. If this should happen, the ID cannot be moved until someone has physically corrected the problem.

The motor drive shutdown chassis contains a high-power solid-state relay to which the ac input power to the motor drives is wired. This chassis has a $5 \mathrm{~V}$ power supply wired to four of the minimum limit switches on the ID. This is accomplished by connecting a two-wire cable to the ID interface chassis where these four limit switches are connected in series with each switch wired to the interface chassis in parallel. If any one of these four limit switches is hit, the solidstate relay's $5 \mathrm{~V}$ input is turned off, thus turning off the ac input power to the motor drives.

The board was designed for use with the Oregon Micro Systems (OMS) VME8 - eight-axis intelligent stepper-motor controller. This motor controller has step, direction, and auxiliary outputs as well as limit switch inputs on the P2 connector of the VME bus. The P2 connector connects to the ID control card through a 64-conductor ribbon cable. The axis select register on the ID control card is read/write register that allows selection of any two of the eight axes to control the stepper motors. Once the bits in this register have been set, they are decoded by custom decoder logic to decode the step, direction, auxiliary, and limit signals to and from the motor controller, thus allowing axis selection at any time.

Standard EPICS motor record and device support ${ }^{3}$ (version 3.5 and up) allows the use of pre- and postmove commands to the motor record using the motor controller's command set. The auxiliary line for a particular axis is asserted before the move starts and deasserted after the move stops. These lines are used to change the output power level of the motor drives being used, if they are so equipped. This allows the motor drive to be set to provide a lower constant current to the motor when the device is not in motion, thereby increasing the motor life and reliability. The stepper-motor drives that are being used at the APS for these two motor insertion devices are manufactured by Superior Electric and are 12 A stepper-motor drives that have both boost and reduce inputs.

These inputs allow us to set the motor drive current to 8 $\mathrm{A}$, and when the ID is not moving, the drive current is reduced by $50 \%$ (to only 4 A). When the ID starts moving, boost is asserted causing the motor drive to boost the current by $50 \%$ (up to $12 \mathrm{~A}$ ). All motor drive control and feedback signals are located on the front panel using two DB9 connectors, one connector for each drive. These particular motor drives also have an output that tells when the drive is ready to move motors. This output is fed back to the ID control card and gives us the ability to remotely see if the drives are ready.

The digital inputs and outputs (DIOs) are located on one of the 40-pin condo header connectors on the front panel. There are two read/write registers that are used for the 16 bits of digital outputs and one more read-only register that is used for the digital inputs. The DIO can be used for anything that requires 5 or $0 \mathrm{~V}$ and less than $20 \mathrm{~mA}$ of drive current. All digital outputs may be read back through the VME bus to provide a means of knowing which bits have been set or reset.

The ID interlock registers contain bits that allow the system manager to disable the ID motion remotely using standard EPICS records. This same register also contains bits for device hardware monitoring such as emergency stop buttons and control signals for the motor drives. There are two readonly bits, one for each motor drive. These bits provide feedback as to whether or not the motor drives are ready to move motors. An adjustable voltage-to-frequency converter on the control card provides a pulse train to the stepper-motor drives for the purpose of an emergency open. The emergency open can be asserted remotely using EPICS, which opens the ID regardless of any other commands that have been issued. The ID will continue to open until at least one of the maximum limit switches has been hit, an emergency stop button is pressed, or the key switch on the front panel has been 
turned. There is also an external input from the front-end equipment protection system (FEEPS) that can also assert the emergency open signal.

The encoder interface is an eight-channel chassis specifically designed as an interface to the Varoc synchronous serial interface (SSI) VME card from the European Synchrotron Radiation Facility. The rear panel contains eight circular Molex connectors each having eight pins that are wired to a D-subminiature 50-pin connector (DB50). The chassis also contains two power supplies, one $5 \mathrm{~V}$ that supplies power to a set of linear encoders and one $15 \mathrm{~V}$ that supplies power to a set of rotary encoders. There are two 50-pin header connectors on the front panel of the Varoc SSI board, each with eight encoder channels. This is the reason for the encoder interface chassis, as each chassis may be easily connected to the Varoc card using a mass terminated 50-conductor ribbon cable.

\section{TESTING}

Software has been written to automate testing of the ID control VME and the ID interface boards. This will not only ease the burden of testing but also ensure more complete and consistent test results. The boards' digital inputs and outputs are used in conjunction with special test cables connected to the ID interface card that allow the VME board to be self- testing. Control signal outputs from the card are fed back into the digital inputs and then checked for accuracy. Only the motor controller's step pulse train and the on-board voltage-to-frequency converter must be manually tested.

\section{CONCLUSIONS}

The APS ID control system has been in production since July 1999. It has been successfully used for control and diagnostics of both a single ID and two IDs at the same beamline. This control system has proven to be a very reliable and versatile design.

\section{ACKNOWLEDGMENTS}

The authors would like to thank Bob Laird for his continued hardware support and Frank Lenkszus for the development of the VME test software. This work is supported by the U.S. Department of Energy, Office of Basic Energy Sciences, under Contract No. W-31-109-ENG-38.

\footnotetext{
${ }^{1}$ M. Ramanathan, M. Smith, J. Grimmer, and M. Merritt, Rev. Sci. Instrum. 73, 1448 (2002).

${ }^{2}$ J. Grimmer, M. Ramanathan, M. Smith, and M. Maerritt, Rev. Sci. Instrum. 73, 1433 (2002).

${ }^{3}$ J. Kowalkowski, T. Mooney, J. Sullivan, and R. Sluiter (private communication).
} 